\title{
A Convex Optimization Approach to Feedback Scheduling
}

\author{
Mongi Ben Gaid, Daniel Simon and Olivier Sename
}

\begin{abstract}
Adaptive tasks scheduling via feedback is an effective solution for the optimization of closed-loop control performance under computing power limitation, as in the computing nodes of embedded networked control systems. The problem of the feedback scheduling of a set of Linear-Quadratic controllers, based on both plant-state and resource utilization measurements is considered. The proposed feedback scheduler acts on tasks periods in order to minimize a quadratic cost functional over an infinite horizon, for the overall system, and to achieve a desired processor utilization. Based on KarushKuhn-Tucker conditions, it is shown that if Riccati matrix coefficients may be approximated using parabolic functions of the sampling frequency, the expressions of optimal sampling frequencies may be computed analytically. Examples where these approximations hold are presented and the approach is illustrated on a simulation example.
\end{abstract}

\section{INTRODUCTION}

A constant challenge in embedded systems development is represented by computational resource limitations. In fact, economic constraints impose to realize the desired functionalities with the lowest cost. These limitations call for a more efficient use of the available resources. In this context, integrated control and scheduling methodologies have been proposed in order to allow a more flexible and efficient utilization of the computational resources [1].

The problem of optimal sampling period selection, subject to schedulability constraints, was first introduced in [2]. Considering a bubble control system benchmark, the relationship between the control cost (corresponding to a step response) and the sampling periods were approximated using convex exponential functions. Using the Karush-KuhnTucker (KKT) first order optimality conditions, the analytic expressions of the optimal off-line sampling periods were established. The problem of the joint optimization of control and off-line scheduling has been studied in [3], [4], [5].

The idea of feedback scheduling was introduced in [6], [7]. First approaches in feedback scheduling considered feedback from resource utilization (for example tasks execution times) in order to optimize the control performance [6], [8], or to minimize a deadline miss ratio in soft real-time systems [7]. Naturally, the online adjustment of sampling periods calls for optimal sampling periods assignment. The approaches in [6], [8] used a similar method to [2] in order find analytic expressions of the optimal sampling periods, under cost approximation assumptions (linear or quadratic approximation

This work was supported by the French National Research Agency (ANR) project Safe-NeCS under grant ANR-05-SSIA-0015-03

M. Ben Gaid and D. Simon are with the NeCS Project-Team, INRIA Rhône-Alpes, Montbonnot Saint Martin, France (e-mails: \{Mohamed.Bengaid, Daniel.Simon\}@inrialpes.fr). O. Sename is with the Control Systems Department, Gipsa-Lab, INPG-CNRS UMR 5216 Saint Martin d'Hères, France (e-mail: olivier.sename@inpg.fr). of the cost as a function of the sampling period). The experimental evaluation of the feedback scheduling concept was undertaken in [9]. The issue of guaranteeing the stability and performance of the controlled systems, when their sampling periods are varied on-line (by a feedback scheduler, for example), was addressed in [10], using the $H_{\infty}$ approach for linear parameter varying systems.

Later, it was pointed out that the optimal sampling frequencies are also dependent on the controlled system actual state [11], [12], and not only on off-line considerations. The problem of the optimal integrated control and scheduling was formalized (using a hybrid systems approach) and solved in [13]. Heuristics for integrated control and non-preemptive scheduling were proposed, in particular the OPP [13] and RPP [5] algorithms as well as the relaxed dynamic programming based scheduling strategy [14]. A common point to these heuristics is that the scheduling decisions (which task to execute or message to send) are determined on-line through the comparison of a finite number of quadratic functions of the extend state (actual state extended by previous controls). These quadratic cost functions are pre-computed off-line based on the intrinsic characteristics of the controlled systems. Another common point is that concurrency was modeled in a finely grained way. Related approaches were proposed in [15], and where scheduling decisions are based on the discrepancies between current and the most recently transmitted values of nodes' signals. These latter results may be applied to the problem of dynamic scheduling of CAN networks.

Other approaches relied on the notion of periodic task, and used task periods as scheduling decision modification variable. In particular, in [16], the problem of the optimal sampling period selection of a set of LQG controllers, based on plant states knowledge, was studied. It has been shown that the optimal solution to this problem is too complicated. The optimal LQG cost, as a function of the sampling period, was depicted for some selected numerical examples. Explicit formulas, relating the optimal sampling periods to the plant state were derived in the case of the minimum variance control of first order plants. The issue of the choice of the feedback scheduler period was also studied. The same setting was considered in [17]. The on-line sampling period assignment was based on a look-up table, which was constructed off-line, for predefined values of the sampling periods. A heuristic procedure, allowing the construction of this lookup table was also proposed.

Other approaches of state-based resource allocation were proposed [18], [19]. Although these approaches do not target to optimize a global cost function, their objective is to 
allocate the computational resources in order to achieve other control objectives such as the asymptotic stability [18] or a specified $l_{2}$ attenuation level [19].

However, all the previously cited approaches for statefeedback optimal scheduling did not exploit or investigate the possible convexity properties of the optimal state-feedback based resource allocation problem. In fact, in these approches, the solutions were based on rather generic optimization methods or special heuristics. However, in optimization, convexity is an interesting property that considerably simplifies the obtained solutions. Convex optimization problems have in general efficient solutions. In the context of the on-line state-feedback scheduling, the complexity of the scheduling algorithm is a crucial point for its effectiveness.

The ideas developed in this paper may be seen as the generalization of the approaches of [2], [6], [8] to the context of optimal state-feedback scheduling of $N$ LQ controllers with variable sampling periods. Instead of approximating the cost as a function of the sampling period, we suggest a sampling-period approximation of the different coefficients of the Riccati equations solutions, which are involved in the LQ control design. We illustrate some examples where this approximation holds. We present then the algorithm allowing the computation of the optimal state-dependant sampling periods. Finally, simulation results illustrate the proposed approach.

\section{PROBLEM FORMULATION}

Consider a collection of $N$ continuous-time LTI systems $\left\{\mathscr{S}_{i}\right\}_{1 \leq i \leq N}$. Each system $\mathscr{S}_{i}$ is described by the state space representation

$$
\dot{x}_{i}(t)=A_{i} x_{i}(t)+B_{i} u_{i}(t),
$$

where $x_{i} \in \mathbb{R}^{n_{i}}$ and $u_{i} \in \mathbb{R}^{m_{i}}$. An infinite horizon continuoustime cost functional $J_{i}$, defined by

$$
J_{i}\left(x_{i}, u_{i}\right)=\int_{0}^{\infty}\left(x_{i}^{T}(t) Q_{i} x_{i}(t)+u_{i}^{T}(t) R_{i} u_{i}(t)\right) d t,
$$

is associated to $\mathscr{S}_{i}$, and represents the design specifications of its ideal controller. We assume that $Q_{i}$ and $R_{i}$ are positive definite matrices of appropriate dimensions and that the pair $\left(A_{i}, B_{i}\right)$ is reachable. Each system $\mathscr{S}_{i}$ is controlled by a control task $\tau_{i}$, characterized by a period $h_{i}$ and an executiontime $C_{i}$. These two parameters may be time-varying. The $N$ control tasks $\left\{\tau_{i}\right\}_{1 \leq i \leq N}$ are executed on the same processor. A global cost functional $J\left(x_{1}, \ldots, x_{N}, u_{1}, \ldots, u_{N}\right)$, defined by

$$
J\left(x_{1}, \ldots, x_{N}, u_{1}, \ldots, u_{N}\right)=\sum_{i=1}^{N} \omega_{i} J_{i}\left(x_{i}, u_{i}\right),
$$

is associated to the entire system, allowing the evaluation of its global performance. Constants $\left\{\omega_{i}\right\}_{1 \leq i \leq N}$ are weighting factors, representing the relative importance of each control loop.

The main objective of this paper is to design a feedback scheduler, allowing assigning tasks periods $\left\{h_{i}\right\}_{1 \leq i \leq N}$ that optimize the global control performance (defined by $J$ ), subject to processor utilization constraints (defined by $U_{s p}$ ), and based on both tasks execution time $\left\{C_{i}\right\}_{1<i<N}$ and plants states measurements $\left\{x_{i}\right\}_{1 \leq i \leq N}$, as shown in Fig. 1.

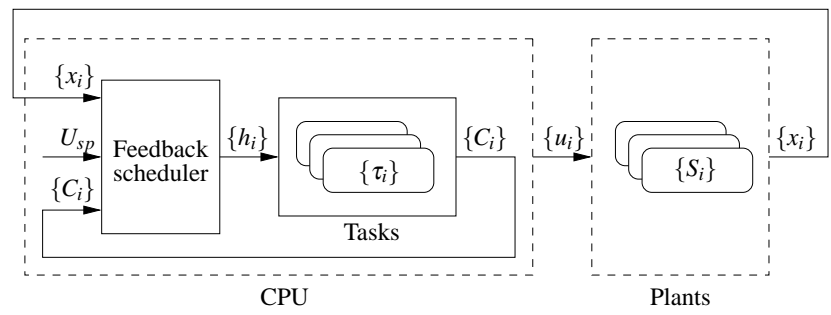

Fig. 1. Integrated plant-state and execution-time feedback scheduling

Remark 1: $U_{s p}$ represents the desired processor utilization of tasks whose periods are controlled by the feedback scheduler. It may be chosen by the designer in order to cope with the presence of other tasks, whose processor utilization is not controlled by the feedback scheduler. In practice, even in the situations where all the tasks are controlled by the feedback scheduler, choosing $U_{s p}$ less than the schedulable utilization bound allows to obtain a "utilization margin" and to avoid the overruns that may result from the variations of tasks execution times.

\section{COST FUNCTIONS DEFINITION AND APPROXIMATION}

\section{A. Cost functions definition}

For a given fixed sampling period $h_{i}$ of system $\mathscr{S}_{i}$, assume that there exists an optimal sampled-data controller $u_{i, h_{i}}^{*}$, defined by the state-feedback control gain $K_{h_{i}}^{*}$, and which minimizes the cost functional (2), subject to plant model (1) and to zero-order hold constraints

$$
u_{i, h_{i}}(t)=u_{i, h_{i}}\left(k h_{i}\right) \quad \text { for } k h_{i} \leq t<(k+1) h_{i} .
$$

The expression of $K_{h_{i}}^{*}$ may be found in control textbooks, for example [20]. The computation of $K_{h_{i}}^{*}$ requires the solving of an algebraic Riccati equation (ARE).

Let $t_{i}(k)$ be the $k^{\text {th }}$ instant where the control input $u_{i}$ is updated and

$$
J_{i}\left(t_{i}(k), x_{i}, u_{i, h_{i}}^{*}\right)=\int_{t_{i}(k)}^{\infty}\left(x_{i}^{T}(t) Q_{i} x_{i}(t)+u_{i, h_{i}}^{*^{T}}(t) R_{i} u_{i, h_{i}}^{*}(t)\right) d t .
$$

An interesting property in optimal LQ sampled-data control is that the cost functional $J_{i}\left(t_{i}(k), x_{i}, u_{i, h_{i}}^{*}\right)$ may be characterized by a unique positive definite matrix $S_{i}\left(h_{i}\right)$ of size $n_{i} \times n_{i}$, which is the solution of the ARE. This property considerably simplifies the computation of cost function (5), when the optimal sampled-data control $u_{i, h_{i}}^{*}$ is used. In fact, instead of simulating the evolution of the sampled-data system (1)(4) and using equation (5) for cost computation, it suffices to use the formula

$$
J_{i}\left(t_{i}(k), x_{i}, u_{i, h_{i}}^{*}\right)=x_{i}\left(t_{i}(k)\right)^{T} S_{i}\left(h_{i}\right) x_{i}\left(t_{i}(k)\right) .
$$

In the following, we will denote by $S^{c}$ the solution of the ARE associated to the problem of finding the optimal continous time controller $u_{i, c}^{*}$, which minimizes the cost functional (2), subject to plant dynamics (1). The quality of control (QoC) measure, associated to each system, will be the 
difference between the optimal sampled-data cost $J_{i}\left(x_{i}, u_{i, h_{i}}^{*}\right)$ and the optimal continous-time cost $J_{i}\left(x_{i}, u_{i, c}^{*}\right)$.

$$
\begin{aligned}
J_{i}^{*}\left(x_{i}(0), h_{i}\right) & =J_{i}\left(x_{i}, u_{i, h_{i}}^{*}\right)-J_{i}\left(x_{i}, u_{i, c}^{*}\right) \\
& =x_{i}(0)^{T}\left(S_{i}\left(h_{i}\right)-S_{i}^{c}\right) x_{i}(0)
\end{aligned}
$$

and similarly

$$
\begin{aligned}
J_{i}^{*}\left(x_{i}\left(t_{i}(k)\right), h_{i}\right) & =J_{i}\left(t_{i}(k), x_{i}, u_{i, h_{i}}^{*}\right)-J_{i}\left(t_{i}(k), x_{i}, u_{i, c}^{*}\right) \\
& =x_{i}\left(t_{i}(k)\right)^{T}\left(S_{i}\left(h_{i}\right)-S_{i}^{c}\right) x_{i}\left(t_{i}(k)\right)
\end{aligned}
$$

\section{B. An introductory example}

Consider the linearized model of an unstable second order pendulum, described by

$$
\dot{x}(t)=A x(t)+B u(t),
$$

with $A=\left[\begin{array}{cc}0 & 1 \\ -\frac{g}{l} & -\frac{f_{v}}{m l^{2}}\end{array}\right], B=\left[\begin{array}{c}0 \\ \frac{1}{m l^{2}}\end{array}\right]$ and $x(t)=\left[\begin{array}{c}\theta(t) \\ \dot{\theta}(t)\end{array}\right]$, where $\theta(t)$ represents the pendulum angle, $l$ its length, $m$ its weight, $f_{v}$ the viscous friction coefficient, $g$ the gravitational acceleration and $u(t)$ the control input. The numerical values of these parameters are $l=1, m=1, f_{v}=1$, and $g=9.81$. The design specifications are described by the weighting matrices $Q=\operatorname{Diag}(100,10)$ and $R=0.05$.

The blue '+' marks in Fig. 2 represent the values of the different coefficients of matrix $\left(S(h)-S^{c}\right)$, as a function of the sampling period. It easy to see that the coefficients of $\left(S(h)-S^{c}\right)$ may be approximated as a parabolic function of $h$. The green curve in Fig. 2 represent the mean square best fitting parabola of $\left(S(h)-S^{c}\right)$. Using a basic linear least square method, $S(h)-S^{c}$ was approximated as

$$
S(h)-S^{c} \approx \Theta h^{2},
$$

where

$$
\Theta=\left[\begin{array}{rr}
69.9541 & 20.9162 \\
20.9162 & 6.2751
\end{array}\right]
$$

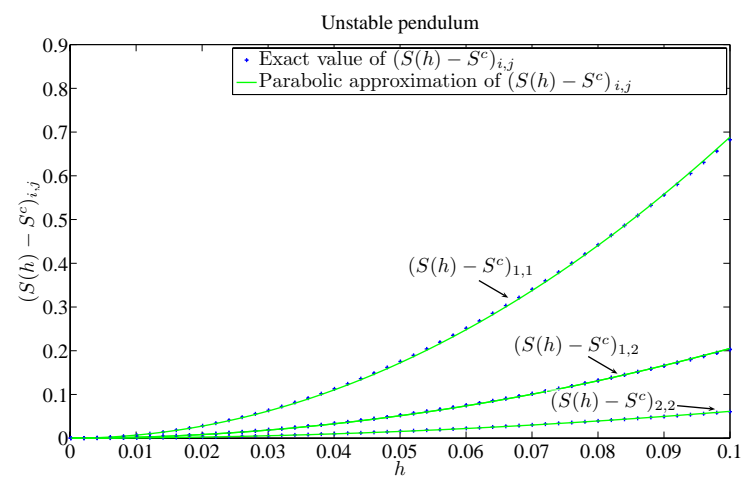

Fig. 2. Unstable pendulum : cost coefficients vs. sampling period

This parabolic evolution of the cost was observed in two other benchmarks : a linearized model of the attitude of a quadrotor helicopter [21] (for $0 \leq h \leq 50 \mathrm{~ms}$ ) and a 14 order car active suspension system [13] (for $0 \leq h \leq 15 \mathrm{~ms}$ ), as illustrated in Figs. 3 and 4.

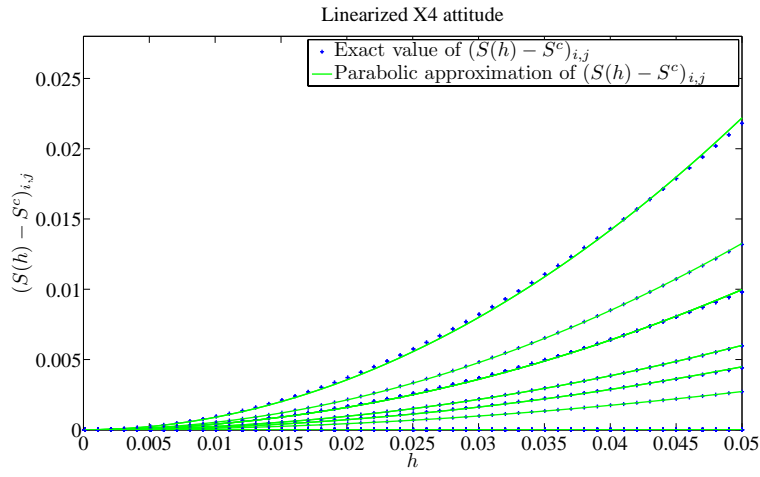

Fig. 3. X4 quadrotor : cost coefficients vs. sampling period

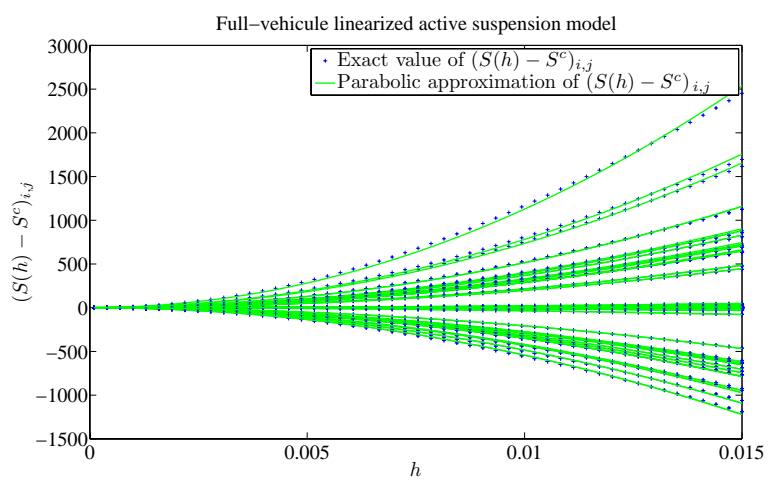

Fig. 4. Active suspension : cost coefficients vs. sampling period

These examples illustrate that in many situations, it is possible to approximate the relationship between the solutions of the Riccati equation and the sampling period, using polynomial interpolations, over a defined range of sampling periods. In the remaining of this paper, we will assume that for $0 \leq h_{i} \leq h_{i}^{\max }$

$$
S_{i}\left(h_{i}\right)-S_{i}^{c} \approx \Theta_{i} h_{i}^{2}
$$

Note that although only this approximation is considered in this paper, the obtained results may be easily generalized to other polynomial approximations.

Remark 2: The choice of $h_{i}^{\max }$ depends on the quality and the validity of approximating the true values of Riccati matrix coefficient using parabolic functions.

Remark 3: Based on Riccati equation solutions approximations, analytic expressions of the control gains as a function of the sampling period may be easily deduced.

Remark 4: The relationship between the cost function and the sampling frequencies may become more complicated, and even non convex, when the frequencies are decreased near the Nyquist rate, as illustrated in [6].

\section{OPTIMAL SAMPLING PERIOD SELECTION}

\section{A. Problem formulation}

Let $f_{i}=\frac{1}{h_{i}}$ be the sampling frequency (corresponding to the sampling period $h_{i}$ ). Assume that $C_{i}$ are constant (we will show in the next subsection how the time-variations of 
$C_{i}$ may be handled). The optimal sampling period frequency selection problem may be formulated as follows.

$$
\left\{\begin{array}{l}
\min _{f_{1}, \ldots, f_{N}} \sum_{i=1}^{N} \omega_{i} \frac{x_{i}^{T} \Theta_{i} x_{i}}{f_{i}^{2}} \\
\text { subject to: } \\
\sum_{i=1}^{N} C_{i} f_{i} \leq U_{s p} \\
f_{i} \geq f_{i}^{\text {min }} \text { for } i=1, \ldots, N
\end{array}\right.
$$

where $f_{i}^{\text {min }}=\frac{1}{h_{i}^{\max } \text {. }}$

Remark 5: In optimization problem (7), the values of the sampling frequencies are implicitly upper bounded by the processor utilization constraint. Furthermore, it is also straightforward to add upper bound constraints on the sampling frequencies (i.e. by adding constraints $f_{i} \leq f_{i}^{\max }$ or equivalently $h_{i}^{\text {min }} \leq h_{i}$ ). However, these additional constraints may lead to a slight complexification of the problem resolution.

\section{B. Problem solving}

Problem (7) has a convex objective function and affine inequality constraints. Consequently, if the feasibility region is non empty, its optimal solution will exist, and may be computed analytically using the Karush-Kuhn-Tucker (KKT) conditions [22]. The analysis of the different conditions of KKT conditions leads to the following algorithm for the computation of the optimal sampling frequencies.

Compute $\beta_{i}=\omega_{i} x_{i}^{T} \Theta_{i} x_{i}$

Compute $\Gamma_{i}=\frac{2 \beta_{i}}{C_{i} f_{i}^{\min ^{3}}}$;

Sort $\Gamma_{i}$ in increasing order (i.e. find the permutation $\varphi$ such that $\left.\Gamma_{\varphi(1)} \leq \Gamma_{\varphi(2)} \leq \ldots \leq \Gamma_{\varphi(N)}\right)$;

Determine the largest integer $p$ such that

$$
\begin{aligned}
& \sum_{i=1}^{p} C_{\varphi(i)} f_{\varphi(i)}^{\min }+\sum_{i=p+1}^{N} C_{\varphi(i)}\left(\frac{\beta_{\varphi(i)} C_{\varphi(p)}}{\beta_{\varphi(p)} C_{\varphi(i)}}\right)^{\frac{1}{3}} f_{\varphi(p)}^{\min } \geq U_{s p} \\
& \text { if } 1 \leq i \leq p \text { then } \\
& \quad f_{\varphi(i)}^{*}=f_{\varphi(i)}^{\min } ; \\
& \text { endif } \\
& \text { if } p+1 \leq i \leq N \text { then } \\
& \quad f_{\varphi(i)}^{*}=\left(\frac{\beta_{\varphi(i)}}{C_{\varphi(i)}}\right)^{\frac{1}{3}} \frac{U_{s p}-\sum_{j=1}^{p} C_{\varphi(j)} f_{\varphi(j)}^{\min }}{\sum_{j=p+1}^{N} \beta_{\varphi(j)}^{\frac{1}{3}} C_{\varphi(j)}^{\frac{2}{3}}}
\end{aligned}
$$

Algorithm 1: Optimal sampling frequencies computation

Algorithm 1 is a the result of the direct application of KKT conditions. Its proof is given in Appendix.

\section{Feedback scheduling algorithm deployment}

The feedback scheduler is executed as a periodic task, with period $h_{f b s}$. The choice of this period is a tradeoff between the complexity of the feedback scheduler and the performance improvements it brings, as illustrated in [16].

Tasks execution times may be estimated on-line and smoothed using a first-order filter

$$
\hat{C}_{i}\left(k h_{f b s}\right)=\lambda \hat{C}_{i}\left((k-1) h_{f b s}\right)+(1-\lambda) C_{i}\left(k h_{f b s}\right) .
$$

where $\lambda$ is a forgetting factor, $\hat{C}_{i}\left(k h_{f b s}\right)$ and $C_{i}\left(k h_{f b s}\right)$ are respectively the estimated and the measured execution-times at instant $k h_{f b s}$.

In practice, using Algorithm 1, the optimal sampling frequencies of a plant tend to be reduced to zero as the plant approaches the equilibrium. This has the drawback of reducing the disturbance rejection abilities of that plant. Another drawback is that when all the plants approach the equilibrium, coefficients $\beta_{i}$ tend to approach zero. The optimal sampling frequencies assignment may result in an undetermined form 0/0. Fortunately, all these issues may be solved if a constant term, representing "a prediction of the cost of future disturbances" is added to the cost functions 2 . This amounts to replace

$$
\beta_{i}=\omega_{i} x_{i}^{T} \Theta_{i} x_{i}
$$

by

$$
\beta_{i}=\omega_{i} x_{i}^{T} \Theta_{i} x_{i}+\bar{\beta}_{i},
$$

where $\bar{\beta}_{i}$ are constants coefficient, which have to chosen offline, according to the future disturbances that a given plant may be subjected to. These coefficients may be chosen by trial and error, until the best behavior is obtained. A small value of $\bar{\beta}_{i}$ increases the sensibility of the optimal sampling period $h_{i}^{*}$ with respect to state values. A larger value reduces this state sensibility.

Remark 6: The expression of $\bar{\beta}_{i}$ may be explicitly computed if a linear quadratic Gaussian formulation and a finite optimization horizon are adopted in the optimal sampling frequency assignment problem (instead of the deterministic infinite horizon formulation that was adopted in this paper).

\section{Simulation Results}

In order to illustrate the proposed feedback scheduling approach, we consider two unstable pendulums, as described in Section III-B. Each pendulum $i(i \in\{1,2\})$ is controlled by a task $\tau_{i}$. Tasks execution times are equal to $C_{1}=C_{2}=20 \mathrm{~ms}$. The desired utilization of these two tasks is $U_{s p}=80 \%$. Tasks periods $h_{1}$ and $h_{2}$ that may be assigned by the feedback scheduling algorithm verify

$$
0 \leq h_{1} \leq h_{1}^{\max } \text { and } 0 \leq h_{2} \leq h_{2}^{\max },
$$

where $h_{1}^{\max }=h_{2}^{\max }=300 \mathrm{~ms}$. Constants $\bar{\beta}_{1}$ and $\bar{\beta}_{2}$ are equal to $10^{-3}$. The period of the feedback scheduler is $h_{f b s}=$ $150 \mathrm{~ms}$.

In these simulations, pendulum 1 is disturbed by a rectangular signal of period $T=2 \mathrm{~s}$, pulse width $\alpha=0.15$, offset $o=20 \mathrm{~ms}$ and with random amplitude $a(k), k \in \mathbb{N}$, verifying a uniform distribution in $[-10,10]$. This disturbance signal is described by

$$
w(t)= \begin{cases}a(k) & \text { if } k T+o \leq t<(k+\alpha) T+o \\ 0 & \text { if }(k+\alpha) T+o \leq t<(k+1) T+o .\end{cases}
$$

Pendulum 2 is disturbed by a band-limited white noise with sampling period $1 \mathrm{~ms}$ and noise power $1 \times 10^{-4}$. The feedback scheduling approach (FBS) is compared to a fixed period scheduling (FPS), where the computational resources 
are fairly distributed between the two control tasks (ie. since the two pendulums are identical, the tasks periods are set to $h_{1}=h_{2}=\frac{U_{s p}}{2 C_{1}}=\frac{U_{s p}}{2 C_{2}}=50 \mathrm{~ms}$ ).
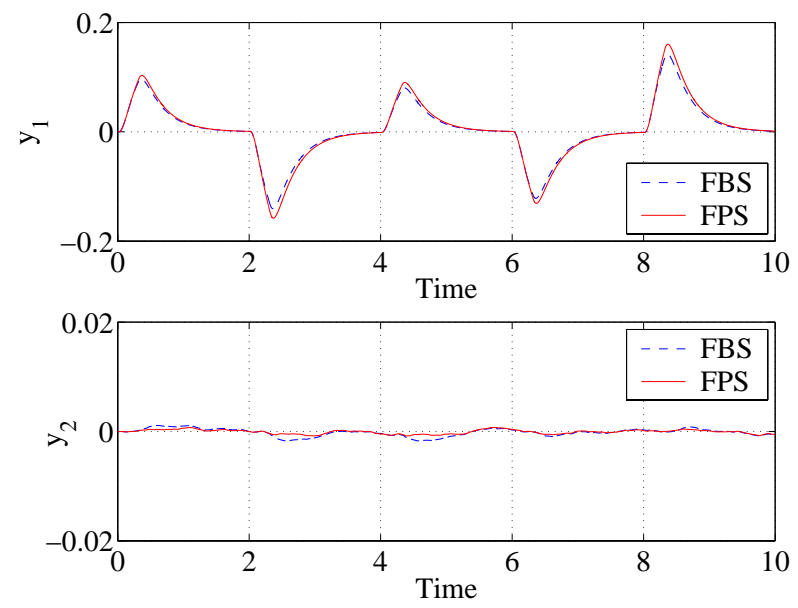

Fig. 5. Pendulums outputs (using fixed-period scheduling (FPS) and feedback scheduling (FBS))

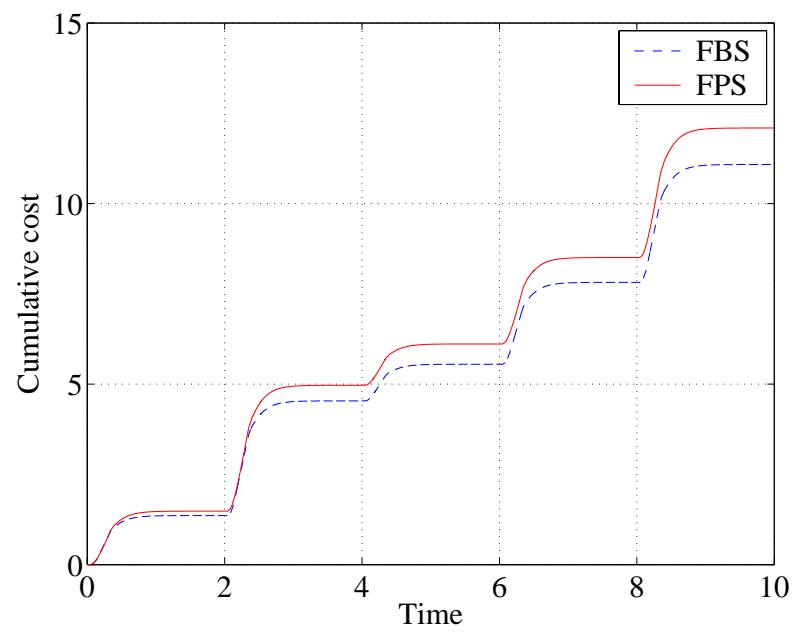

Fig. 6. Cumulative total cost (using fixed-period scheduling (FPS) and feedback scheduling (FBS))

Note that this example, tasks execution times were assumed to be constant. For that reason, a resource based feedback scheduling approach (for example [8]) would have a similar performance to the FPS algorithm.

Pendulums outputs and cumulative cost are depicted in Figs. 5 and 6 . Tasks sampling periods assigned by the feedback scheduler in Fig. 7. These simulations illustrate how the feedback scheduling algorithm reduces the sampling period of the pendulum which is subjected to the most severe disturbance (pendulum 1), in order to improve the global control performance (as measured by the cost function Fig. $6)$. When the disturbance is rejected, the sampling periods are reset to their optimal offline values $(50 \mathrm{~ms})$. Although performance improvements which are observed in this particular example are not spectacular, the use of the feedback
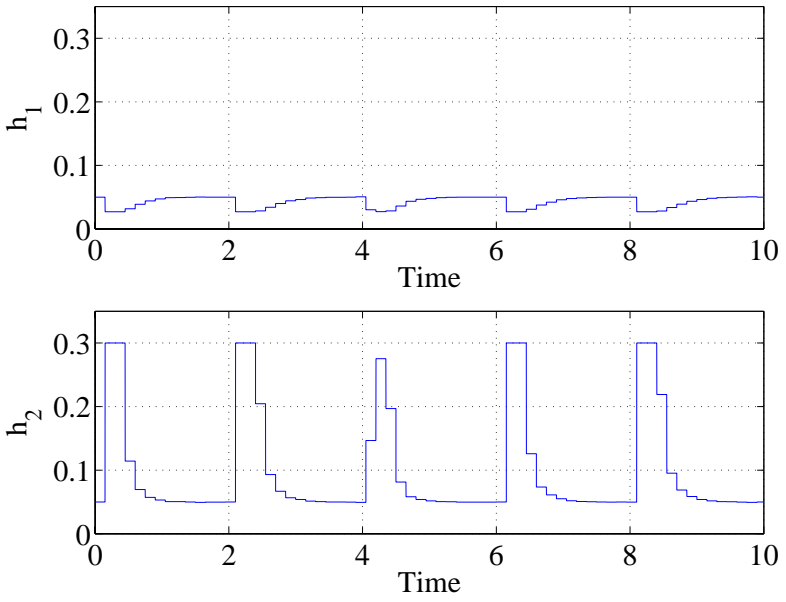

Fig. 7. Tasks $\tau_{1}$ and $\tau_{2}$ periods (using feedback scheduling (FBS))

scheduling approach induces robustness improvements which are not directly observable on the control performance. In fact, as illustrated in [9], feedback scheduling allows improving the robustness with respect to the temporal non determinism of the implementation [23].

\section{CONCLUSIONS}

This paper proposed ideas targeting to simplify the complexity (in terms of computation and memory) of the problem of optimal on-line sampling frequency assignment, through the full exploitation of its convexity properties. The main simplification comes from the approximation of Riccati matrix coefficients, as a function of the sampling frequency. The proposed strategy was applied using a feedback scheduling scheme, and evaluated by simulation.

In this paper, the control performance was measured using a Linear Quadratic cost. Future work aims at generalizing the proposed ideas to the problems of optimal sampling period assignment in the sense of the $H_{\infty}$ performance criterion. Future work will also include the real-time implementation of the proposed approach on platforms where execution resources are time-varying as well, allowing taking into account the overhead of the feedback scheduling algorithm and the uncertainties coming from the implementation.

\section{REFERENCES}

[1] K.-E. Årzén, A. Cervin, J. Eker, and L. Sha, "An introduction to control and real-time scheduling co-design," in Proceedings of the 39th IEEE Conference on Decision and Control, Sydney, Australia, December 2000.

[2] D. Seto, J. P. Lehoczky, L. Sha, and K. G. Shin, "On task schedulability in real-time control systems," in Proceedings of the 17th IEEE RealTime Systems Symposium, New York, USA, December 1996.

[3] H. Rehbinder and M. Sanfridson, "Scheduling of a limited communication channel for optimal control," Automatica, vol. 40, no. 3, pp. 491-500, March 2004.

[4] B. Lincoln and B. Bernhardsson, "LQR optimization of linear system switching," IEEE Transactions on Automatic Control, vol. 47, no. 10, pp. 1701-1705, October 2002.

[5] M.-M. Ben Gaid, A. Çela, Y. Hamam, and C. Ionete, "Optimal scheduling of control tasks with state feedback resource allocation," in Proceedings of the 2006 American Control Conference, Minneapolis, Minnesota, USA, June 2006. 
[6] J. Eker, P. Hagander, and K.-E. Årzén, "A feedback scheduler for realtime control tasks," Control Engineering Practice, vol. 8, no. 12, pp. 1369-1378, December 2000.

[7] C. Lu, J. Stankovic, G. Tao, and S. Son, "Feedback control real-time scheduling: Framework, modeling and algorithms," Special issue of Real-Time Systems Journal on Control-Theoretic Approaches to RealTime Computing, vol. 23, no. 1/2, pp. 85-126, Jul/Sept 2002.

[8] A. Cervin, J. Eker, B. Bernhardsson, and K.-E. Årzén, "Feedbackfeedforward scheduling of control tasks," Real-Time Systems, vol. 23, no. 1, pp. 25-53, July 2002.

[9] D. Simon, D. Robert, and O. Sename, "Robust control / scheduling codesign: application to robot control," in Proceedings of the 11th IEEE Real-Time and Embedded Technology and Applications Symposium, San Francisco, USA, March 2005.

[10] D. Robert, O. Sename, and D. Simon, "A reduced polytopic LPV synthesis for a sampling varying controller : experimentation with a T inverted pendulum," in Proceedings of the European Control Conference, Kos, Greece, July 2007.

[11] P. Martí, J. Fuertes, G. Fohler, and K. Ramamritham, "Improving quality-of-control using flexible timing constraint : Metric and scheduling issues," in Proceedings of the 23rd IEEE Real-Time Systems Symposium, Austin, Texas, USA, December 2002.

[12] P. Martí, C. Lin, S. Brandt, M. Velasco, and J. Fuertes, "Optimal state feedback based resource allocation for resource-constrained control tasks," in Proceedings of the 25th IEEE Real-Time Systems Symposium, Lisbon, Portugal, December 2004.

[13] M.-M. Ben Gaid, A. Çela, and Y. Hamam, "Optimal integrated control and scheduling of networked control systems with communication constraints: Application to a car suspension system," IEEE Transactions on Control Systems Technology, vol. 14, no. 4, pp. 776-787, July 2006.

[14] A. Cervin and P. Alriksson, "Optimal on-line scheduling of multiple control tasks: A case study," in Proceedings of the 18th Euromicro Conference on Real-Time Systems, Dresden, Germany, July 2006.

[15] D. B. Dačić and D. Nešić, "Quadratic stabilization of linear networked control systems via simultaneous protocol and controller design," Automatica, vol. 43, no. 7, pp. 1145-1155, 2007.

[16] D. Henriksson and A. Cervin, "Optimal on-line sampling period assignment for real-time control tasks based on plant state information," in Proceedings of the Joint 44th IEEE Conference on Decision and Control and European Control Conference, Seville, Spain, December 2005.

[17] R. Castañé, P. Martí, M. Velasco, A. Cervin, and D. Henriksson, "Resource management for control tasks based on the transient dynamics of closed-loop systems," in Proceedings of the 18th Euromicro Conference on Real-Time Systems, Dresden, Germany, July 2006.

[18] P. Tabuada, "Event-triggered real-time scheduling of stabilizing control tasks," IEEE Transactions on Automatic Control, vol. 52, no. 9, pp. 1680-1685, 2007.

[19] M. Lemmon, T. Chantem, X. Hu, and M. Zyskowski, "On selftriggered full information h-infinity controllers," in Proceedings of Hybrid Systems: Computation and Control, April 2007.

[20] K. J. Åström and B. Wittenmark, Computer-controlled systems: theory and design. Prentice Hall, 1997.

[21] J. F. Guerrero-Castellanos, A. Hably, N. Marchand, and S. Lesecq, "Bounded attitude stabilization: Application on four-rotor helicopter," in Proceedings of the 2007 IEEE International Conference on Robotics and Automation, Roma, Italy, April 2007.

[22] S. P. Boyd and L. Vandenberghe, Convex optimization. Cambridge University Press, 2004.

[23] A. Cervin, D. Henriksson, B. Lincoln, J. Eker, and K.-E. Årzén, "How does control timing affect performance?" IEEE Control Systems Magazine, vol. 23, no. 3, pp. 16-30, June 2003.

\section{APPENDIX}

To simplify the proof, and without loss of generality, assume that $\Gamma_{i}, i \in\{1, \ldots, N\}$, are arranged in increasing order. Assume also that there exists $i \in\{1, \ldots, N\}$ such that $\beta_{i} \neq 0$ (otherwise, we will have a trivial situation where any feasible solution is also optimal).

We first introduce Lagrangian multipliers $\lambda_{i}, i \in\{1, \ldots, N\}$ and $\lambda$. Let $\bar{f}=\left[f_{1}, \ldots, f_{N}\right]^{T}$ and $\bar{\lambda}=\left[\lambda_{1}, \ldots, \lambda_{N}\right]^{T}$. The
Lagrangian of optimization problem (7) is defined by

$$
\mathscr{L}(\bar{f}, \bar{\lambda}, \lambda)=\sum_{i=1}^{N} \frac{\beta_{i}}{f_{i}^{2}}+\lambda\left(\sum_{i=1}^{N} C_{i} f_{i}-U_{s p}\right)+\sum_{i=1}^{N} \lambda_{i}\left(f_{i}^{\text {min }}-f_{i}\right) .
$$

The KKT conditions express the necessary conditions that the solution of the problem, as well as the Lagrangian multipliers, shall satisfy at the optimum, and are described by

$$
\begin{aligned}
\frac{\partial \mathscr{L}}{\partial f_{i}}\left(\bar{f}^{*}, \bar{\lambda}^{*}, \lambda^{*}\right) & =0 \\
\lambda^{*}\left(\sum_{i=1}^{N} C_{i} f_{i}^{*}-U_{s p}\right) & =0 \\
\lambda_{i}^{*}\left(f_{i}^{\text {min }}-f_{i}^{*}\right) & =0 \\
\sum_{i=1}^{N} C_{i} f_{i}^{*}-U_{s p} & \leq 0 \\
f_{i}^{\text {min }}-f_{i}^{*} & \leq 0 \\
\lambda^{*} & \geq 0 \\
\lambda_{i}^{*} & \geq 0
\end{aligned}
$$

Condition $\frac{\partial \mathscr{L}}{\partial f_{i}}\left(\bar{f}^{*}, \bar{\lambda}^{*}, \lambda^{*}\right)=0$ is equivalent to

$$
-2 \frac{\beta_{i}}{f_{i}^{*^{3}}}+\lambda^{*} C_{i}-\lambda_{i}^{*}=0 \text { for } i \in\{1, \ldots, N\} .
$$

Since $\lambda_{i} \geq 0$, and there exists $i$ such that $\beta_{i}>0$, then $\lambda>0$ and $\sum_{i=1}^{N} C_{i} f_{i}^{*}=U_{s p}$. This means that at the optimum, all the sampling frequencies are assigned in a way that achieves the maximal desired utilization. In order to determine the other constraints that are active at the optimum, we first let $f_{i}=$ $f_{i}^{\text {min }}$, for $i \in\{1, \ldots, N\}$. We start increasing the frequencies, beginning with $f_{N}$. It is easy to see that for each $f_{i}>f_{i}^{\min }$, we have $\lambda_{i}=0$, and consequently

$$
\lambda=\frac{2 \beta_{i}}{C_{i} f_{i}^{3}}
$$

This last relation shows that $\lambda$ decreases as $f_{i}$ increases. In order for equation (9) to hold for $\lambda_{i} \geq 0$, it is necessary that each $f_{i}$ starts increasing from $f_{i}^{\text {min }}$ when $\lambda<\Gamma_{i}$. To determine which frequencies $f_{i}$ are equal or greater than $f_{i}^{\text {min }}$, it is necessary to find the lagrest integer $p$ such that

$$
\sum_{i=1}^{p-1} C_{i} f_{i}^{m i n}+C_{p} f_{p}^{m i n}+\sum_{i=p+1}^{N} C_{i}\left(\frac{2 \beta_{i}}{C_{i} \lambda}\right)^{\frac{1}{3}} \geq U_{s p}
$$

with $\lambda=\Gamma_{p}$. This last expression is equivalent to

$$
\sum_{i=1}^{p} C_{i} f_{i}^{\min }+\sum_{i=p+1}^{N} C_{i}\left(\frac{\beta_{i}}{\beta_{p}} \frac{C_{p}}{C_{i}}\right)^{\frac{1}{3}} f_{p}^{\min } \geq U_{s p}
$$

Finally, we conclude that the optimal sampling frequencies assignment is defined by

$$
\begin{cases}f_{i}^{*}=f_{i}^{\text {min }} & \text { for } i=1, \ldots, p, \\ f_{i}^{*}=\left(\frac{\beta_{i}}{C_{i}}\right)^{\frac{1}{3}} \frac{U_{s p}-\sum_{j=1}^{p} C_{j} f_{j}^{\min }}{\sum_{j=p+1}^{N} \beta_{j}^{\frac{1}{3}} C_{j}^{\frac{2}{3}}} & \text { for } i=p+1, \ldots, N .\end{cases}
$$

